\title{
MYOCARDIAL INFARCTION AND ISCHEMIC HEART DISEASE IN PATIENTS WITH ACUTE ISCHEMIC STROKE
}

\author{
Daniela Y. Arabadzhieva, \\ Zhaneta T. Georgieva', \\ Ara G. Kaprelyan, \\ Zdravko D. Slavov ${ }^{2}$ \\ First Clinic of Neurology, \\ ${ }^{1}$ Department of Propedeutics of \\ Internal Medicine, \\ St. Marina University Hospital of \\ Varna \\ ${ }^{2}$ Chernorizets Hrabar Varna Free \\ University, Varna
}

\author{
Corresponding Author: \\ Daniela Y.Arabadzhieva \\ First Clinic of Neurology \\ St. Marina University Hospital of Varna \\ 1, Hristo Smirnenski blvd. \\ Varna, 9010 \\ Bulgaria \\ e-mail:d.arabadzhieva@abv.bg
}

Received: October 29, 2014

Revision received: January 09, 2015

Accepted: May 18, 2015

\begin{abstract}
Summary
The aim of the investigation was to analyze the correlation between myocardial infarction and ischemic heart disease, on the one hand, and acute ischemic stroke (AIS), on the other hand. We studied 258 AIS patients (mean age $70.9 \pm 7.22$ years, range $49-92$ years) hospitalized in 2007 2013 in the First Clinic of Neurology, St. Marina University Hospital of Varna. The diagnosis of acute ischemic stroke was confirmed by Doppler sonography and computed tomography of the cerebral circulation. Data were statistically processed by variation and correlation analysis. Our results proved a relatively strong correlation between effort angina pectoris and ischemic heart disease (Pearson's coefficient: $\mathrm{r}=0.643$ ) as well as a weak correlation between ischemic heart disease and myocardial infarction (Pearson's coefficient: $\mathrm{r}=0.243$ ) among acute ischemic stroke patients. The well-known risk factors for these cardiovascular diseases such as obesity, tobacco smoking, low physical activity and alcohol abuse were common among the patients with acute ischemic stroke, too. In conclusion, both myocardial infarction and ischemic heart disease are strongly associated with the development of acute ischemic stroke. Such patients require strict and regular control by general practitioners. They should observe an appropriate diet and adhere to a healthy life-style.
\end{abstract}

Key words: acute ischemic stroke, myocardial infarction, ischemic heart disease, correlation analysis

\section{Introduction}

Ischemic stroke (IS) after myocardial infarction (MI) or ischemic heart disease (IHD) is an important complication. The occurrence of cerebrovascular accident in MI patients is associated with increased morbidity and mortality. On the other hand, patients with transient ischemic attack and IS are at an increased risk for subsequent cardiac events, including MI. Rapid identification of IS patients at higher risk for MI might foster intensified cardiac monitoring or certain therapeutic strategies. IS has emerged as a new coronary artery disease (CAD) risk equivalent. Modern secondary prevention of the acute IS (AIS) benefits more from epidemiologic 
studies focusing on risk factor modifications, rather than antiplatelet or other AIS specific agents.

The aim of this investigation was to analyze the correlation between MI and IHD, on the one hand, and AIS, on the other hand.

\section{Materials and Methods}

We studied 258 AIS patients (mean age $70.9 \pm 7.22$ years, range $49-92$ years) hospitalized in 2007-2013 in the First Clinic of Neurology, St. Marina University Hospital of Varna. There were 129 males aged 69.60 \pm 7.80 years (range 49-87 years) and 129 females aged $71.59 \pm 6.65$ years (range 58-92 years). The diagnosis of AIS was confirmed by Doppler sonography and computed tomography of the cerebral circulation. The presence of three cardiovascular diseases was analyzed: IHD, MI and effort angina pectoris (AP). Data were statistically processed by variation (ANOVA as $t$-criterion was considered significant if $\mathrm{p}<0.05$ ) analysis and correlation (Pearson's coefficient) analysis as well. SPSS, version 13.0 software was applied.

\section{Results}

Table 1 shows patient distribution by age.

Table 2 illustrates the correlation dependences between IHD, AP and MI among our AIS patients. We established a relatively strong correlation between AP and IHD as well as a weak correlation between IHD and MI among AIS. In addition, the well-known risk factors for these cardiovascular diseases such as obesity, tobacco smoking, low physical activity and alcohol abuse were common among the patients with AIS, too (data not shown).

\section{Discussion}

There are certain most recent foreign publications dealing with the interactions of MI and IHD with AIS.

A total of 173233 unselected acute MI (AMI) patients from the Swedish Register of Information and Knowledge about Swedish Heart Intensive Care Admissions registry for 1998-2008 were analyzed [1]. The one-year mortality was $36.5 \%$ for AMI complicated by IS and $18.3 \%$ for AMI without IS. It decreased from 1998 to 2008 in patients with and without IS. The absolute mortality rates decreased by $9.4 \%$ and $7.5 \%$, respectively, and were associated with reperfusion and secondary preventive therapies. The increased use of evidence-based therapies explained the improved prognosis. This Register was merged with the Swedish National Patient Register [2]. Of 173233 patients with AMI, 3571 (2.06\% of the cases) developed IS within 30 days [3] but 7185 (4.14\% of the cases) had an IS within one year [2]. Percutaneous coronary intervention, fibrinolysis, acetylsalicylic acid, statins, and P2Y12 inhibitors were predictors of reduced risk of IS [3]. There was a $20 \%$ relative risk reduction in 1998-2000 versus 2007-2008 $(p<0.001)$ [2]. Independent predictors of IS were age, female sex, ST-segment-elevation AMI, previous IS, previous diabetes mellitus, heart failure at admission, ACE inhibitor treatment and atrial fibrillation [2,3]. Between 2003 and 2010, of 118434 AMI patients, 40679 had chronic kidney disease (CKD) [4]. An in-hospital IS

Table 1. Age distribution of AIS patients

\begin{tabular}{|c|c|c|c|c|c|c|}
\hline \multirow{2}{*}{ Age (years) } & \multicolumn{2}{|c|}{ males } & \multicolumn{2}{|c|}{ females } & \multicolumn{2}{|c|}{ total } \\
\hline & $\mathrm{n}$ & $\%$ & $\mathrm{n}$ & $\%$ & $\mathrm{n}$ & $\%$ \\
\hline$\leqslant 60$ & 8 & 6.20 & 6 & 4.65 & 14 & 5.43 \\
\hline $61-65$ & 33 & 25.58 & 21 & 16.28 & 54 & 20.93 \\
\hline $66-70$ & 36 & 27.91 & 27 & 20.93 & 63 & 24.42 \\
\hline $71-75$ & 22 & 17.05 & 36 & 27.91 & 58 & 22.48 \\
\hline $76-80$ & 19 & 14.73 & 27 & 20.93 & 46 & 17.83 \\
\hline$\geqslant 81$ & 11 & 8.53 & 12 & 9.30 & 23 & 8.91 \\
\hline total & 129 & 100.00 & 129 & 100.00 & 258 & 100.00 \\
\hline
\end{tabular}


Table 2. Correlations between ischemic heart disease (IHD), effort angina pectoris (AP) and myocardial infarction (MI) in 258 AIS patients

\begin{tabular}{lccc}
\hline & IHD & AP & MI \\
\hline IHD & 1 & 0.643 & 0.243 \\
\hline AP & 0.643 & 1 & 0.073 \\
\hline MI & 0.243 & 0.073 & 1 \\
\hline
\end{tabular}

occurred in 2.3 and $1.2 \%$ of CKD and non-CKD patients, respectively. IS occurrence after hospital discharge decreased between 2003-2004 and 2009-2010 from 4.1 to $2.5 \%$ in CKD patients and from 2.0 to $1.3 \%$ in non-CKD patients, respectively.

During a hospital-based, retrospective and single centre study, 192 consecutive IS patients divided into a subgroup of 54 patients without IHD (55.6\% males) aged $63.1 \pm 11.8$ years and a subgroup of 138 patients with IHD (39.1\% males) aged $76.3 \pm 9.6$ years, the following risk factors were examined: age, gender, body mass index, presence of arterial hypertension (AH) and carotid plaques, atrial fibrillation, diabetes mellitus, as well as plasma levels of total cholesterol, triglycerides, LDL-cholesterol and HDL-cholesterol [5]. The logistic regression analysis demonstrated that only age $(\mathrm{OR}=1.109$; 95\% CI 1.069-1.150; $\mathrm{p}=0.001)$ and the presence of $\mathrm{AH} \quad(\mathrm{OR}=6.298 ; 95 \%$ CI 2.215-17.905; $\mathrm{p}=0.003$ ) were exclusively and significantly associated with IHD presence in IS patients.

In an observational study from the Nationwide Inpatient Sample in the USA for 2006-2008, the overall rate of IS among the 1924413 patients admitted for AMI was $1.47 \%$ [6]. In the prospective Austrian Stroke Unit Registry over a six-year period (January 1, 2007 to January 13, 2013), 421 of 41619 transient ischemic attack/IS patients $(1.01 \%$ of the cases) presented with MI during treatment for a median duration of three days [7]. These patients were significantly older; clinically more severely affected and had more frequently vascular risk factors, atrial fibrillation and previous MI. They not only suffered from worse short-term outcome including a higher mortality $(14.5$ versus $2 \% ; \mathrm{p}<0.001)$ at stroke unit discharge, but also acquired more stroke complications like progressive stroke and pneumonia.

Among 33503 diabetes mellitus patients, IS incidence rate during the first year after AMI common complication after an AMI in diabetes mellitus patients, but this risk recently decreased due to the increased use of evidence-based therapies.

Some 45 relevant studies that were identified from PubMed, EMBASE and four Chinese databases up to July 2013 covered a total of 7238 cases and 7395 controls [9]. The $\beta$-fibrinogen$455 \mathrm{G} / \mathrm{A}$ polymorphism was associated with the risk of IS when compared with the dominant model $(\mathrm{OR}=1.518,95 \%$ CI $1.279-1.802$ for $\mathrm{AA}+\mathrm{GA}$ versus $\mathrm{GG}$ ) as well as of CAD $(\mathrm{OR}=1.802,95 \% \mathrm{CI}=1.445-2.246)$.

In the course of a prospective, nested casecontrol study, the associations between high sensitivity-C-reactive protein (hs-CRP) and risks of cardiovascular disease within a cohort of 29 876 Japanese men and women aged 40-69 years were examined [10]. There were 638 IS patients and $168 \mathrm{MI}$ patients. A linear association between hs-CRP levels and risks of IS and MI $(p=0.07)$ was observed. The predictive value of hs-CRP for IS was reduced after adjustment for hypertensive status and body mass index.

Coronary calcium score (CCS) was significantly higher in IS patients than in the control group $(p<0.0001)$ [11]. High-risk CAD $(\mathrm{CCS} \geqslant 400)$ was detected in $24.5 \%$ of IS patients and in $9.3 \%$ of the control group $(p<0.0001)$. In a multiple logistic regression analysis, age, diabetes mellitus, IS and male sex were significantly associated with high-risk CAD $(p<0.001)$.

The association between the acyl-CoA: cholesterol acyltransferase-1 (ACAT-1) single nucleotide polymorphism rs1044925, on the one hand, and the risk of CAD and IS, on the other hand, was studied in $587 \mathrm{CAD}$ and 555 IS patients, as well as in 588 controls [12]. The $\mathrm{C}$ allele carriers of ACAT-1 rs1044925 were associated with a decreased risk in CAD and IS patients, and an increased serum HDLcholesterol level in the healthy controls.

The investigation of 159 consecutive AIS patients who were living independently before AIS and were diagnosed with CAD before AIS by using pre-stroke CHADS, CHA2DS2-VASc and R2CHADS2 scores demonstrated that these tools were useful in predicting the functional status and poor outcome after AIS in CAD patients [13]. A reperfusion strategy for simultaneous MI with ST-segment elevation and AIS within a time window was suggested [14].

Pre-year stroke sodium valproate treatment $(28 \%)$ was associated with increased IS risk 
(odds ratio $1.22,95 \%$ CI $1.09-1.38, \mathrm{p}<0.001$ ) while there was no association between ever being prescribed sodium valproate with IS (odds ratio $1.01,95 \%$ CI $0.91-1.12, p=0.875$ ) [15]. A significant association was observed between ever being prescribed sodium valproate with MI (odds ratio $0.78,95 \%$ CI $0.67-0.90, \mathrm{p}<0.001$ ). Development of new medications for treating acute or chronic IHD may provide opportunity to cross over into IS. These drugs include desmoteplase, tenecteplase, tirofiban, prasugrel, and ticagrelor [16].

\section{Conclusions}

In conclusion, both myocardial infarction and ischemic heart disease are strongly associated with the development of acute ischemic stroke. Such patients require strict and regular control by the general practitioners. They should observe an appropriate diet and adhere to a healthy life-style.

\section{References}

1. Brammås A, Jakobsson S, Ulvenstam A, Mooe T. Mortality after ischemic stroke in patients with acute myocardial infarction: predictors and trends over time in Sweden. Stroke. 2013;44(11):3050-5.

2. Ulvenstam A, Kajermo U, Modica A, Jernberg T, Söderström L, Mooe T. Incidence, trends, and predictors of ischemic stroke 1 year after an acute myocardial infarction. Stroke. 2014;45(11):32638.

3. Kajermo U, Ulvenstam A, Modica A, Jernberg T, Mooe T. Incidence, trends, and predictors of ischemic stroke 30 days after an acute myocardial infarction. Stroke. 2014;45(5):1324-30.

4. Jakobsson S, Graipe A, Huber D, Björklund F, Mooe T. The risk of ischemic stroke after an acute myocardial infarction in patients with decreased renal function. Cerebrovasc Dis. 2014;37(6):4609.

5. Kovacik M, Madarasz S, Kral M, Veverka T, Herzig R, Kanovsky P. Risk factors associated with ischemic heart disease occurence in acute ischemic stroke patients. Biomed Pap Med Fac Univ Palacky Olomouc Czech Repub. 2013;157(2):168-71.

6. Naderi N, Masoomi H, Mozaffar T, Malik S. Patient characteristics and comorbidities associated with cerebrovascular accident following acute myocardial infarction in the United States. Int J Cardiol. 2014;175(2):323-7.
7. Gattringer T, Niederkorn K, Seyfang L, SeifertHeld T, Simmet N, Ferrari J, et al. Myocardial infarction as a complication in acute stroke: results from the Austrian stroke unit registry. Cerebrovasc Dis. 2014;37(2):147-52.

8. Jakobsson S, Bergström L, Björklund F, Jernberg T, Söderström L, Mooe T. Risk of ischemic stroke after an acute myocardial infarction in patients with diabetes mellitus. Circ Cardiovasc Qual Outcomes. 2014;7(1):95-101.

9. Gu L, Liu W, Yan Y, Su L, Wu G, Liang B, et al. Influence of the $\beta$-fibrinogen-455G/A polymorphism on development of ischemic stroke and coronary heart disease. Thromb Res. 2014;133(6):993-1005.

10. Iso H, Noda H, Ikeda A, Yamagishi K, Inoue M, Iwasaki M, et al. The impact of C-reactive protein on risk of stroke, stroke subtypes, and ischemic heart disease in middle-aged Japanese: the Japan public health center-based study. J Atheroscler Thromb. 2012;19(8):756-66.

11. Iwasaki K, Haraoka K, Hamaguchi T, Imamura T, Kawada S, Ohno M, et al. Prevalence of subclinical coronary artery disease in ischemic stroke patients. J Cardiol. 2015;65(1):71-5.

12.Wu DF, Yin RX, Cao XL, Chen WX. Association between single nucleotide polymorphism rs 1044925 and the risk of coronary artery disease and ischemic stroke. Int $\mathrm{J}$ Mol Sci. 2014;15(3):3546-59.

13. Hoshino T, Ishizuka K, Shimizu S, Uchiyama S. CHADS2, CHA2DS2-VASc, and R2CHADS2 scores are associated with 3-month functional outcome of stroke in patients with prior coronary artery disease. Circ J. 2014;78(6):1481-5.

14.González-Pacheco H, Méndez-Domínguez A, Vieyra-Herrera G, Azar-Manzur F, MeaveGonzález A, Rodríguez-Zanella $\mathrm{H}$, et al. Reperfusion strategy for simultaneous STsegment elevation myocardial infarction and acute ischemic stroke within a time window. Am J Emerg Med. 2014;32(9):1157.e1-4.

15.Dregan A, Charlton J, Wolfe CD, Gulliford MC, Markus HS. Is sodium valproate, an HDAC inhibitor, associated with reduced risk of stroke and myocardial infarction? A nested case-control study. Pharmacoepidemiol Drug Saf. 2014;23(7):759-67.

16. Thomas K, Kessler C. New antiplatelet agents prescribed to patients with ischemic heart disease: implications for treatment of stroke. Curr Treat Options Neurol. 2014;16(5):289. 